\title{
Morphometry of the internal capsule on MR images in adult healthy individuals
}

\author{
Ozan Turamanlar $^{1}$ (D), Abdülkadir Bilir ${ }^{1}$ (D), Erdal Horata ${ }^{2}$ (D), Tolga Ertekin ${ }^{1}$ (D), \\ Çiğdem Özer Gökaslan ${ }^{3}$ (D), Hazal Emeksiz ${ }^{4}$ (D) \\ ${ }^{1}$ Department of Anatomy, Faculty of Medicine, Afyonkarahisar Health Sciences University, Afyonkarahisar, Turkey \\ ${ }^{2}$ Atatiirk Vocational School of Health Services, Afyonkarabisar Health Sciences University, Afyonkarabisar, Turkey \\ ${ }^{3}$ Department of Radiology, Faculty of Medicine, Afyonkarabisar Health Sciences University, Afyonkarahisar, Turkey \\ ${ }^{4}$ Department of Physiotherapy and Rebabilitation, Faculty of Health Sciences, Afyonkarahisar Health Sciences University, Afyonkarabisar, Turkey
}

\begin{abstract}
Objectives: There is substantial information on the morphometric differences of the pathways passing through the internal capsule according to the dominant extremity; however, the diameters of the internal capsule in the horizontal plane have not been previously evaluated. The aim of this study was to evaluate the diameters of parts of the internal capsule (anterior limb, posterior limb and genu) and angle in between these parts in healthy subjects.
\end{abstract}

Methods: MRI images of 80 females and 37 males (age: 18-65) with no obvious intracranial pathology were evaluated. The diameters of the anterior and posterior limb and the genu of the internal capsule and the angle between the anterior and posterior limbs were measured.

Results: There was no statistically significant difference in measurements of internal capsule when compared bilaterally in all individuals ( $p>0.05)$. The right and left genu angles were significantly wider in females. This angle in the present study was found as $122^{\circ}$, while the classical knowledge reveals it as around $90^{\circ}$.

Conclusion: Understanding the normal morphometry of this region may help clinicians in the diagnosis and follow-up of some neurological diseases. Some morphometric characteristics of this region have shown differences from the classical knowledge. Further studies in larger samples should be done for re-evaluating the normal ranges of these morphometric values.

Keywords: internal capsule; morphometry; MRI

Anatomy 2020;14(1):49-52 @2020 Turkish Society of Anatomy and Clinical Anatomy (TSACA)

\section{Introduction}

Internal capsule is a white matter consisting of efferent and afferent fibers in the shape of a fan extending vertically to connect some of the cortical centers with the spinal cord. Internal capsule is divided into anterior limb, genu, posterior limb, retrolentiform limb (retrolenticular part) and sublentiform limb (sublenticular part). It is located in the inferomedial part of each cerebral hemisphere and separates the caudate nucleus and thalamus from the lentiform nucleus. The descending and ascending pathways passing through the internal capsule connect the brain hemispheres and subcortical structures to brain stem and spinal cord. ${ }^{[1,2]}$

The parts of the internal capsule carry different descending and ascending axonal pathways which have critical functions. ${ }^{[1]}$ The anterior limb extends between the head of caudate nucleus and the lentiform nucleus. The posterior limb is between the thalamus and the lentiform nucleus. ${ }^{[3]}$

The anterior limb of the internal capsule carries thalamic and brain stem fibers from prefrontal cortical regions that are associated with different aspects of emotion, moti-

This study was a poster presentation at the 20th National Anatomy Congress \& 1st International Mediterranean Anatomy Congress, 6th-9th September 2018, Konya, Turkey. 
vation, cognitive processing and decision-making. ${ }^{[4]}$ The genu portion of the internal capsule contains the corticobulbar tract fibers, which begin from the lower part of the primary motor area and extend to the motor nuclei of the cranial nerves with superior thalamic radiations. ${ }^{[2]}$ The posterior limb of the internal capsule contains posterior thalamic radiations, corticospinal tract, corticorubral tract and corticopontine tract. ${ }^{[1]}$

A decrease in anterior limb volume is associated with psychiatric disorders such as major depressive disorder, bipolar disorder, obsessive-compulsive disorder and schizophrenia. ${ }^{[4]}$ Therefore, knowing the normal morphometry of this region may help clinicians in the diagnosis and follow-up of diseases. In this study, we aimed to evaluate the width of the parts (anterior, posterior and genu) and genu angle of the internal capsule on MR images of healthy individuals.

\section{Materials and Methods}

Our study was performed in Departments of Anatomy and Radiology of Faculty of Medicine at Afyonkarahisar Health Sciences University. This retrospective clinical study was performed after approval of the Clinical Research Ethics Committee of the Afyon Kocatepe University (2018/2-47). MRI images of 80 females and 37 males (age: 18-65) admitted to the Afyon Kocatepe University Hospital with no obvious intracranial pathology were evaluated in this study. Those with pathological findings in white matter (tumors, cysts, bleeding, etc.) and those with head trauma or stroke were not included in the study.

Measurements were made at the level where caudate nucleus, lentiform nucleus and thalamus were seen in the same transverse plane on MRI. Measurements were performed bilaterally from the widest parts of genu, anterior limb, posterior limb of the internal capsule. The angle at the intersection of the line passing through the middle of the anterior and posterior limbs was also measured (Figure 1).

Statistical analysis was performed using SPSS for Windows version 20.0 (SPSS Inc., Chicago, IL, USA). All values were presented as mean \pm standard deviation $(\mathrm{SD})$. The distribution of the data was evaluated by Kolmogorov-Smirnov test. Mann-Whitney U test was used to determine the difference between two groups. Correlation analysis between groups was done using Pearson's correlation test. The results were evaluated at a $95 \%$ confidence interval and $\mathrm{p}<0.05$ was considered statistically significant.

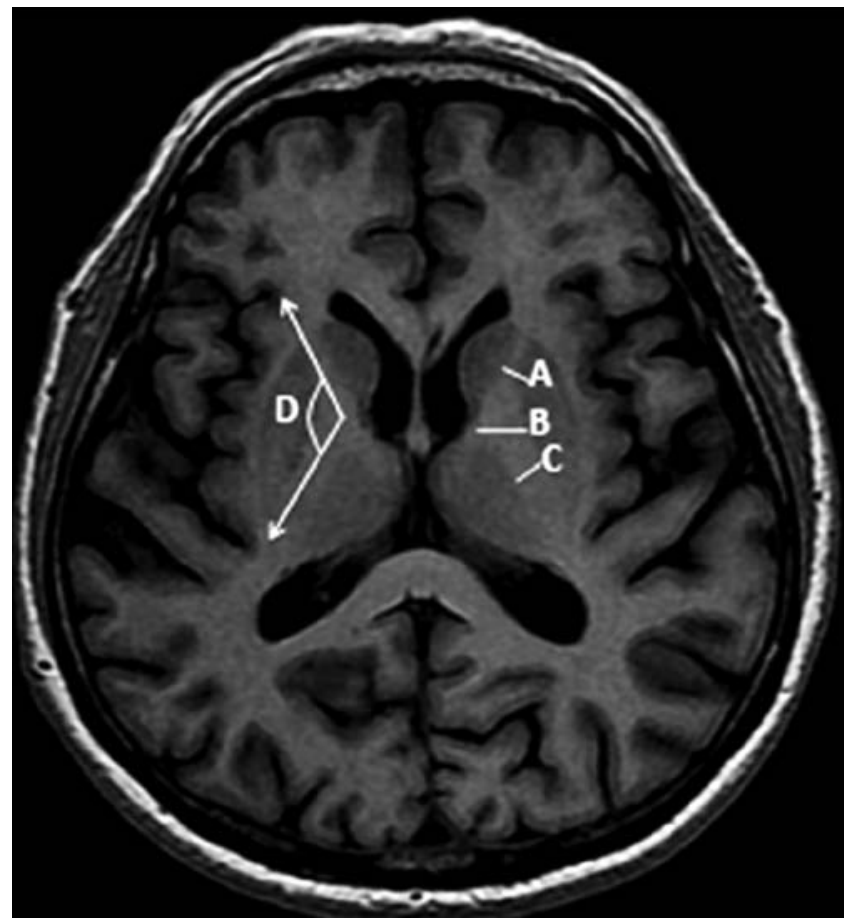

Figure 1. Measurements made in transverse sections. A: width of the anterior limb of internal capsule; B: width of the genu of internal capsule; C: width of the posterior limb of internal capsule; D: genu angle (measured between the lines passing through the middle of the anterior and posterior limbs).

\section{Results}

The mean widths of the anterior limb, genu and posterior limb were measured as $3.05 \pm 0.55 \mathrm{~mm}, 6 \pm 0.62 \mathrm{~mm}$ and $4.23 \pm 0.86 \mathrm{~mm}$ on the left side and $3.12 \pm 0.54 \mathrm{~mm}, 6.05 \pm 0.6$ $\mathrm{mm}$ and $4.04 \pm 0.86 \mathrm{~mm}$ on the right side, respectively. The widths of anterior limb, genu and posterior limb showed no statistically significant difference when compared bilaterally in all individuals $(\mathrm{p}>0.05)$ (Table 1). There was no statistically significant difference between anterior limb, genu, posterior limb measurements among genders as well ( $>0.05)$. However, the right and left genu angles were significantly wider in females than males $(\mathrm{p}<0.05)$. The mean genu angle was measured as $120.58^{\circ} \pm 4.79^{\circ}$ on the left side and $120.53^{\circ} \pm 3.86^{\circ}$ on the right side in males, while it was $123.01^{\circ} \pm 7.69^{\circ}$ on the left side and $123.43^{\circ} \pm 5.12^{\circ}$ on the right side in females (Table 2).

\section{Discussion}

Internal capsule is affected by a variety of pathologies. It is clinically important to know the morphological structure of such a vulnerable site affected by so many diseases (degenerative and demyelinating diseases, vitamin defi- 
Table 1

Comparison of right and left measurements in all subjects $(p<0.05)$.

\begin{tabular}{|c|c|c|c|c|c|}
\hline & \multicolumn{2}{|c|}{ Left } & \multicolumn{2}{|c|}{ Right } & \multirow{2}{*}{$p$-value } \\
\hline & $\min -\max$ & mean $\pm S D$ & $\min -\max$ & mean $\pm S D$ & \\
\hline Anterior limb (mm) & $1.42-4.36$ & $3.05 \pm 0.55$ & $1.96-4.75$ & $3.12 \pm 0.54$ & .273 \\
\hline Genu (mm) & $4.37-7.69$ & $6 \pm 0.62$ & $4.72-7.63$ & $6.05 \pm 0.6$ & .835 \\
\hline Posterior limb (mm) & $2.25-7.12$ & $4.23 \pm 0.86$ & $1.96-6.76$ & $4.04 \pm 0.86$ & .086 \\
\hline Genu angle $(x / 180)$ & $73-137$ & $122.24 \pm 6.98$ & $105.0-135.5$ & $122.51 \pm 4.93$ & .862 \\
\hline
\end{tabular}

ciency, infarction, hemorrhage, arteriovenous malformation, vascular pathologies such as angioma, hypoxia, glioma, oligodendroglioma, ganglioglioma, neoplastic conditions such as neuroectodermal tumor, metastatic lesions, tuberculosis, pyrocystic neurosis, neurogenic cystosis, parasitic diseases such as hydatid cysts, trauma, iatrogenic, epilepsy, psychiatric diseases). ${ }^{[2]}$ There is also need for easily applicable techniques for clinical evaluation and follow-up. It is of great importance to know the morphological features of this anatomical area, which has important functional and clinical features. However, the number of studies evaluating internal capsule morphometry using imaging methods is quite few. Previous studies examining the characteristics of internal capsule relies on findings on cadavers or imaging techniques such as diffusion tensor tractography (DTT) and imaging (DTI), MR and functional MR imaging (fMRI). ${ }^{[-13]}$

DTT provides useful data on localization of the parts of the internal capsule as well as the length or vertical angle of fiber tracts composing the internal capsule. DTT can be used to assess white mater tracts both in healthy individuals and in individuals with pathology (stroke, glioblastoma, multiple sclerosis, amyotrophic lateral sclerosis, etc.). ${ }^{[47,12,13]}$ MRI and FMRI also provides assessment of detecting pathologies affecting the internal capsule. ${ }^{[9,1]}$ In a recent study by Dos Santos et al. ${ }^{[15]}$ brain specimens taken from cadavers stained by a special technique and relationships of the basal nuclei and the internal capsule was described with anatomical coordinates. When all the findings using different imaging techniques compared, it can be seen that the results do not always match.

The genu angle was expressed as $90^{\circ}$ in one of the well-known national anatomy textbook. ${ }^{[16]}$ However, our measurements revealed this angle to be approximately $120^{\circ}$. Considering the difference, we think that this information should be re-evaluated. However, studies with more samples are more likely to yield results that are more accurate.

In conclusion, we suggest that this study on internal capsule morphometry in normal subjects will provide a basis for future studies relying on data regarding internal capsule.

Table 2

Comparison of measurements between genders $(p<0.05)$.

\begin{tabular}{|c|c|c|c|c|c|c|}
\hline & & \multicolumn{2}{|c|}{ Males } & \multicolumn{2}{|c|}{ Females } & \multirow{2}{*}{ p-value } \\
\hline & & $\min -\max$ & mean $\pm S D$ & $\min -\max$ & mean \pm SD & \\
\hline \multirow[t]{4}{*}{ Left } & Anterior limb & $2.14-4.36$ & $3.11 \pm 0.54$ & $1.42-4.19$ & $3.01 \pm 0.55$ & .372 \\
\hline & Genu & $4.48-7.03$ & $5.88 \pm 0.56$ & $4.37-7.69$ & $6.06 \pm 0.64$ & .159 \\
\hline & Posterior limb & $2.71-5.64$ & $4.27 \pm 0.79$ & $2.25-7.12$ & $4.21 \pm 0.89$ & .761 \\
\hline & Genu angle & $111.5-129$ & $120.58 \pm 4.79$ & 73-137 & $123.01 \pm 7.69$ & $.003^{*}$ \\
\hline \multirow[t]{4}{*}{ Right } & Anterior limb & $2.34-4.28$ & $3.14 \pm 0.47$ & $1.96-4.75$ & $3.12 \pm 0.58$ & .813 \\
\hline & Genu & $4.94-7.52$ & $5.91 \pm 0.6$ & $4.72-7.63$ & $6.11 \pm 0.59$ & .086 \\
\hline & Posterior limb & $2.2-5.74$ & $4.06 \pm 0.71$ & $1.96-6.76$ & $4.02 \pm 0.93$ & .831 \\
\hline & Genu angle & $109-126.5$ & $120.53 \pm 3.86$ & $105-135.5$ & $123.43 \pm 5.12$ & $.003^{*}$ \\
\hline
\end{tabular}




\section{Author Contributions}

Concept: OT, AB, EH, TE, ÇÖG; design: OT, AB, EH, TE, ÇÖG; supervision: OT, ÇÖG, TE; data collection \&/or processing: OT, $\mathrm{AB}$, ÇÖG, HE; analysis \&/or interpretation: OT, AB, EH, TE, ÇÖG, HE; literature search: $\mathrm{OT}, \mathrm{AB}, \mathrm{EH}$; writing: OT, $\mathrm{AB}, \mathrm{EH}$; critical review: OT, TE, ÇÖG.

\section{References}

1. Emos MC, Agarwal S. Neuroanatomy, internal capsule. In: StatPearls [Internet]. Treasure Island (FL): StatPearls Publishing; 2019. Available from: https://www.ncbi.nlm.nih.gov/books/NBK542181/

2. Chowdhury F, Haque M, Sarkar M, Ara S, Islam M. White fiber dissection of brain; the internal capsule: a cadaveric study. Turk Neurosurg 2010;20:314-22.

3. Johns P. Clinical neuroscience: an illustrated colour text. Edinburgh: Churchill Livingstone Elsevier; 2014.

4. Safadi Z, Grisot G, Jbabdi S, Tim Behrens T, Heilbronner S, Joe Mandeville J, Versace A, Phillips ML, Lehman JF, Yendiki A, Haber SN. Functional segmentation of the anterior limb of the internal capsule: linking white matter abnormalities to specific connections. J Neurosci 2018;38:2106-17.

5. Domin, M, Langner, S, Hosten, N, Lotze, M. Comparison of parameter threshold combinations for diffusion tensor tractography in chronic stroke patients and healthy subjects. PLoS One 2014;9: e98211.

6. Ferda J, Kastner J, Mukensnabl P, Choc M, Horemuzová J, Ferdová E, Kreuzberg B. Diffusion tensor magnetic resonance imaging of glial brain tumors. Eur J Radiol 2010;74:428-36.

7. Lee DH, Lee DW, Han BS. Topographic organization of motor fibre tracts in the human brain: findings in multiple locations using magnetic resonance diffusion tensor tractography. Eur Radiol 2016; 26:1751-9.

8. Kumar A, Juhasz C, Asano E, Sundaram SK, Makki MI, Chugani DC, Chugani HT. Diffusion tensor imaging study of thecortical origin and course of the corticospinal tract in healthy children. AJNR Am J Neuroradiol 2009;30:1963-70.

9. Qian C, Tan F. Internal capsule: the homunculus distribution in the posterior limb. Brain Behav 2017;7:e00629.

10. Van Hecke W, Nagels G, Leemans A, Vandervliet E, Sijbers J, Parizel PM. Correlation of cognitive dysfunction and diffusion tensor MRI measures in patients with mild and moderate multiple clerosis. J Magn Reson Imaging 2010;31:1492-8.

11. Inal M, Unal B, Kala I, Turkel Y, Bilgili YK. ADC evaluation of the corticospinal tract in multiple sclerosis. Acta Neurol Belg 2015;115: $105-9$.

12. Hecht MJ, Fellner F, Fellner C, Hilz MJ, Heuss D, Neundörfer B. MRI-FLAIR images of the head show corticospinal tract alterations in ALS patients more frequently than T2-, T1- and proton-densityeighted images. J Neurol Sci 2001;186:37-44.

13. Kolasa M, Hakulinen U, Brander A, Hagman S, Dastidar P, Elovaara I, Sumelahti ML. Diffusion tensor imaging and disability progression in multiple sclerosis: a 4-year follow-up study. Brain Behav 2019;9:e01194.

14. Zikou AK, Kitsos G, Tzarouchi LC, Astrakas L, Alexiou GA, Argyropoulou MI. Voxel-based morphometry and diffusion tensor imaging of the optic pathway in primary open-angle glaucoma: a preliminary study. AJNR Am J Neuroradiol 2012;33:128-34.

15. Dos Santos EC, da Luz Veronez DA, de Almeida DB, Piedade GS, Oldoni C, de Meneses MS, Marques MS. Morphometric study of the internal globus pallidus using the Robert, Barnard, and Brown staining method. World Neurosurg 2019;126:e371-78.

16. Arıncı K, Elhan A. Anatomi II. Ankara: Güneş Kitabevi; 2014. p. 313.
ORCID ID:

O. Turamanlar 0000-0002-0785-483X; A. Bilir 0000-0003-0633-9542; E. Horata 0000-0003-1359-228X; T. Ertekin 0000-0003-1756-4366; Ç. Özer Gökaslan 0000-0001-5345-1735; H. Emeksiz 0000-0002-1619-4108
Correspondence to: Ozan Turamanlar MD, PhD Department of Anatomy, Faculty of Medicine, Afyonkarahisar Health Sciences University, Afyonkarahisar, Turkey Phone: +902722463301 e-mail: ozanturamanlar@hotmail.com

Conflict of interest statement: No conflicts declared.

This is an open access article distributed under the terms of the Creative Commons Attribution-NonCommercial-NoDerivs 3.0 Unported (CC BY-NCND3.0) Licence (http://creativecommons.org/licenses/by-nc-nd/3.0/) which permits unrestricted noncommercial use, distribution, and reproduction in any medium, provided the original work is properly cited. Please cite this article as: Turamanlar O, Bilir A, Horata E, Ertekin T, Özer Gökaslan Ç, Emeksiz H. Morphometry of the internal capsule on MR images in adult healthy individuals. Anatomy 2020;14(1):49-52. 